\title{
A HyperCard stack for the creation of graphically organized glossaries
}

\author{
THOMAS A. MARTIN and WALLACE J. GROWNEY \\ Susquehanna University, Selinsgrove, Pennsylvania
}

\begin{abstract}
Many students find it difficult to transform expository text and lectures into well-organized mental constructs. Studies show that learning of this type can be enhanced by the use of graphic organizers that depict relationships among concepts by diagramming them as a tree structure. In order to incorporate all concepts taught in a course into a single graphic organizer, a HyperCard authoring shell was developed to expedite drawing tree diagrams and linking each concept in the diagram to a definition. The authoring features of the stack and its application to an abnormal psychology course are discussed in this article.
\end{abstract}

Instructors often lament the difficulty that students have in transforming expository text and lectures into sets of mental constructs that they can readily recall and apply. A variety of studies have shown that learning of this type can be enhanced by use of graphic organizers (Moore \& Readence, 1984). Graphic organizers identify important concepts and diagram the relationships among them as a tree structure. Although outlines may contain the same hierarchically organized information, Day (1990) has suggested that they are generally inferior to tree diagrams because learners are less likely to remember hierarchical relationships among concepts when information is presented in outline form.

The stack described below expedites the creation of graphically organized glossaries in the HyperCard environment. Impetus for the development of the stack derived from the first author's observation that many students try to learn without putting concepts into any hierarchically organized format, and that even those who outline course material are unlikely to fully master the relationship of one concept to another. It was his wish to incorporate all concepts taught in a course into one graphic organizer, to link each to a definition, and to link key terms in definitions to further definitions. From a practical standpoint, developing such a stack with the basic HyperCard tool set would require an extraordinary investment of time. The obvious solution was to develop an authoring shell that would build graphic organizers from simple lists of concepts.

\section{THE AUTHORING SHELL}

HyperCard 2.1 (Apple Computer, 1991) was used to complete the development of the authoring shell. The stack contains three backgrounds. The first is used for the card

\footnotetext{
The authoring shell described in this paper is available as shareware. Correspondence should be addressed to Thomas Martin, Department of Psychology, Susquehanna University, Selinsgrove, PA 17870 (e-mail:tmartin@einstein.susqu.edu).
}

that welcomes users to the stack. The second background contains objects and scripts that are necessary for the creation and use of graphic organizer cards, and the third contains objects and scripts that are required for building definition cards. The size of each card is $512 \times 342$ pixels, identical to that of the original 9-in. Macintosh screen.

\section{Generating Graphic Organizers}

Graphic organizer cards are generated from a background containing two visible fields, one for the primary concept around which other concepts are organized, and one for all the concepts to be displayed on the card (see field at the bottom of Figure 1). Each concept entered as an item in the second field is converted into a node in the tree diagram, each line of items becomes a row of nodes, and the number of lines determines the number of rows of nodes in the diagram. HyperCard conventions dictate that items are separated by commas, and lines are separated by return characters. Extra commas serve to control the placement of the concept fields by displacing them to the right or left, or generate greater separation between concept fields (see lines 1 and 3 of the field at the bottom of Figure 1).

The drawing of tree diagrams is effected by buttons on the palette displayed in the upper right corner of Figure 1. The first button creates card fields, positions them on the card, inserts one concept per field, and overlays each field with a card button to allow automatic highlighting of the concept when clicked. The second button removes all card buttons and fields so they may be redrawn after changes are made in the background fields. The third button, the paint can icon, fills the card picture with a paint pattern and thereby hides objects in the background. The fourth button, identified by an eraser icon, clears the card of all graphics.

The fifth, sixth, and seventh buttons allow authors to draw lines between concepts to depict their relationship to one another. The author clicks the desired type of line in the palette. Concept fields are then clicked to draw links between them. When drawing is completed, the eighth 


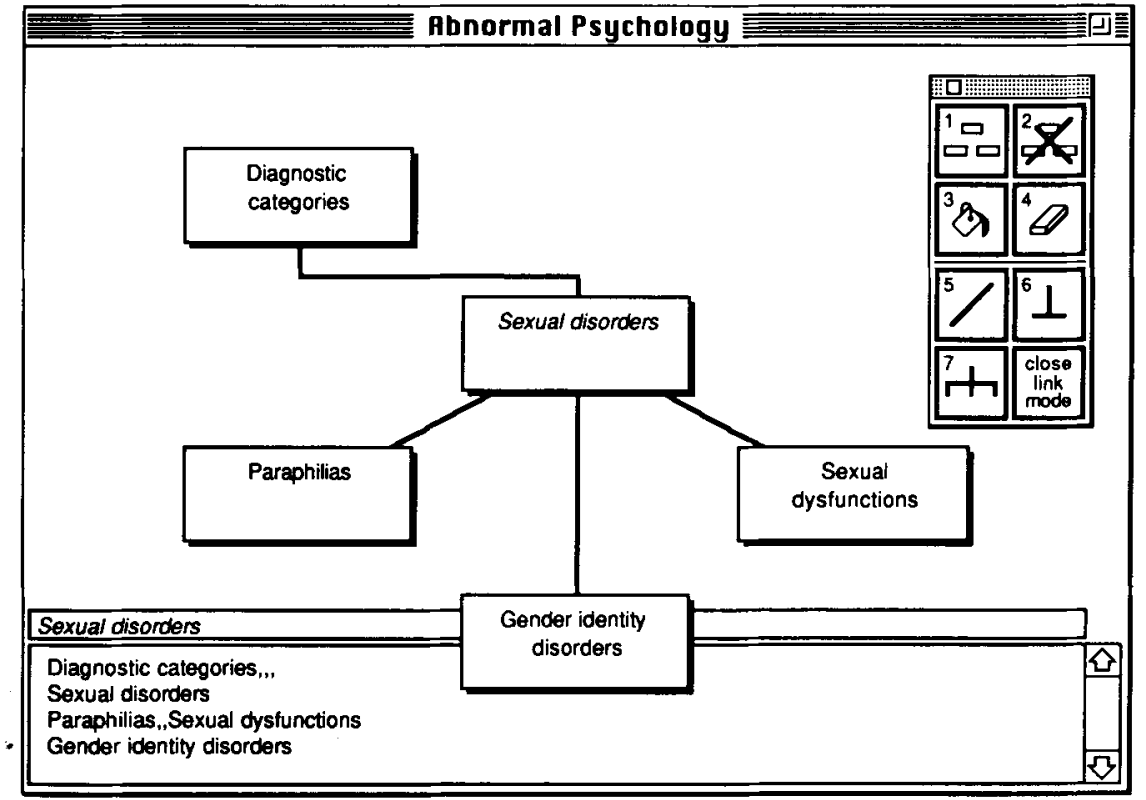

Figure 1. Graphic organizer card with background field and palette visible.

button of the palette must be clicked to close the linkdrawing mode. The result is a graphic organizer card (see Figure 1). The paint icon is then clicked to fill the card picture with a pattern. If the author wishes to change a card or simply look at the information contained in background fields, a command-t keystroke toggles the display between the background and foreground.

Additional graphic organizer cards may be created by clicking a concept field while simultaneously depressing the shift and command keys of the keyboard. The selected concept then becomes the primary concept for the new card. The author may generate the remainder of the tree diagram by typing other concepts into the bottom background field and clicking buttons of the palette. All authoring features are available only if the userLevel of the stack is set to scripting; end users may be denied access to them by restricting the userLevel of the stack to any level below scripting.

The script that creates graphic organizer cards assigns each field a name equivalent to the first 30 characters of the text that it contains. The same script sets the card name to the first 30 characters of the primary concept. Other scripts use these names to control navigation through the stack.

\section{Generating Definition Cards}

Once the graphic organizer exists, authors may generate definition cards by clicking concepts while depressing the shift and command keys of the keyboard. A new definition card is automatically added to the stack, the concept is placed in the small field at the top of the card, and the cursor appears in the larger field to enable entry of the definition. After typing the definition, a command- $t$ keystroke will toggle the lockText property of the field.
Further definition cards may be added for any word or grouped text in a definition by locking the field and then clicking the word or grouped text while depressing the shift and command keys. Again, these authoring features function only when the userLevel of the stack is set to scripting. Clicking the button at the bottom of the screen will take the user back to the card on which the concept is located and lock the definition field if the author has not already done so. Figure 2 shows a completed definition card.

\section{Navigating Through a Stack}

When users click on a concept field, a graphic organizer card with the same name as the field's is displayed. In this way, users can navigate through a large set of hierarchically organized concepts. If there is no graphic organizer card for a concept, or if the command key of the keyboard is depressed while the concept is clicked, the definition of the concept will be shown. On definition cards, words or phrases in bold type signify availability of further definitions. If the user clicks on the bold text, its definition is displayed.

\section{APPLICATION TO A COURSE}

Like many lower division courses in the natural sciences, most abnormal psychology courses are heavily laden with terms and concepts that can be organized into tree diagrams. A common student complaint is that too much material is covered in a given course. From the perspective of the instructor, however, the inability of students to master the material often stems from their failure to organize it adequately. If students treat the concepts as a flat list of terms to memorize, they are likely to learn 


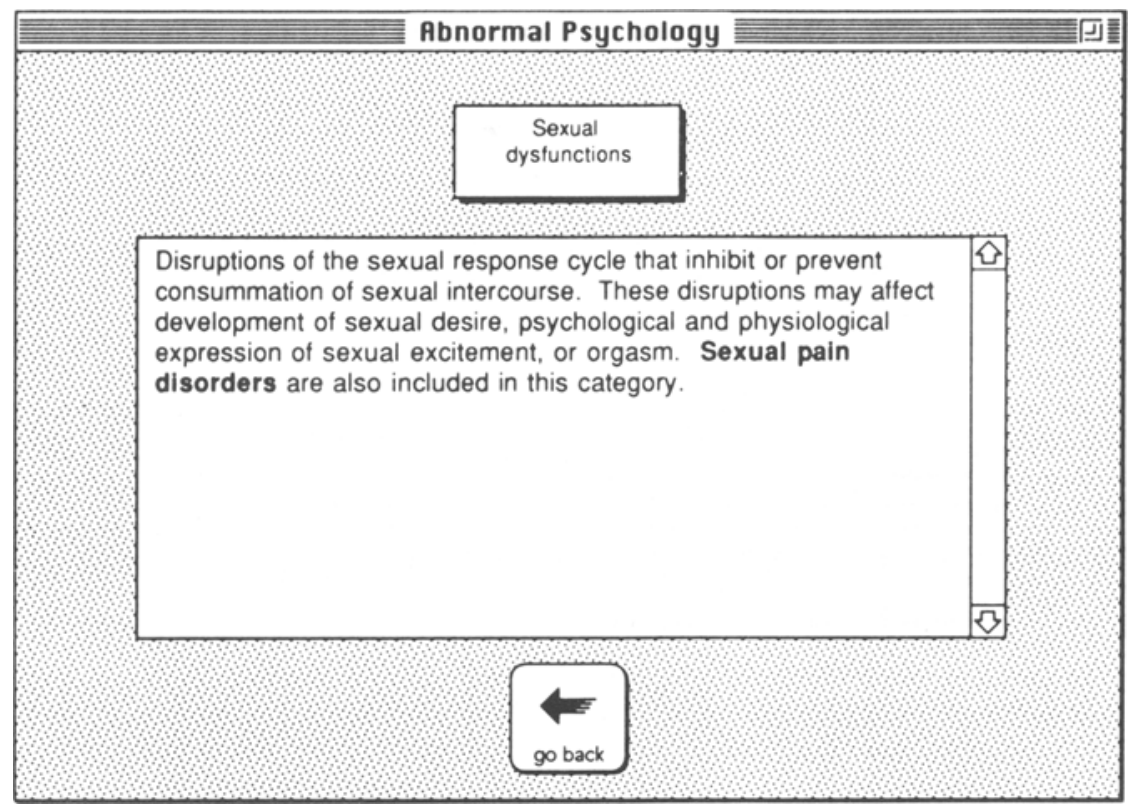

Figure 2. Definition card for sexual dysfunctions.

no more than a small percentage of them. Likewise, superordinate and subordinate relationships share a low probability of retention. Most instructors would prefer that students fully master superordinate concepts and then extend their grasp of the subordinate concepts as far as their ability allows. However, the linear format of both textbooks and lectures makes it difficult to adequately emphasize the advantage of approaching the topic in a hierarchically structured fashion. The central purpose of hypertext applications is to free users from the linearity inherent in traditional media; this characteristic of HyperCard makes it possible to structure an entire course as a set of tree diagrams instead of a linear stream of information.

A project is presently under way to develop a stack to accompany an abnormal psychology course. The course is organized around introductory issues, diagnostic categories, and intervention techniques and issues. These concepts, therefore, are displayed on the first graphic organizer card that students see. When they click on one of the concepts, another graphic organizer card opens which breaks that concept into its subordinate concepts. This process may be repeated until the user reaches a terminus of the tree diagram. For example, navigating from the global concept of abnormal psychology to the definition of multiple-personality disorder is effected by clicking "Diagnostic categories" on the first card, "Dissociative disorders" on the second, and "Multiple personality" on the third to reach the definition.

Each graphic organizer card presents users with a tree diagram that characterizes the relationships among concepts. Figure 3 depicts the diagnostic category of mood disorders. According to the Diagnostic and Statistical Manual of Mental Disorders (American Psychiatric As- sociation, 1987), mood disorders comprise bipolar and depressive disorders. Each of these categories comprises in turn two disorders, one episodic and the other chronic. Major depression and bipolar disorder also occur in a seasonal pattern known as seasonal affective disorder. Users may explore the definition of any of these concepts. They may then retrace their steps by clicking on the "go back" button of the definition cards or the top field of a graphic organizer card.

When the abnormal psychology project is complete, each key concept in the course will be organized in a tree diagram and have a definition associated with it. In essence, the stack will combine the functions of a graphic organizer and a computerized glossary. While it contains far less information than the textbook or classroom experiences, it provides a means of stressing relationships among concepts presented in the course.

\section{Implementation}

Since the abnormal psychology stack remains under development, it was not formally incorporated into the design of the course during the present semester. However, the portion of the stack that is complete (covering approximately $75 \%$ of the course) was installed on five Macintosh SEs in a small computer lab located within the Department of Psychology. In this form, it is available for use by students enrolled in the course.

Evaluations of the stack by students were solicited by an anonymous survey. Of the 26 surveys distributed, 25 were returned. Eight students had used the software; only 1 said it was not useful. The rest indicated that they found it useful primarily as a device for reviewing prior to exams. Their comments were generally positive, includ- 


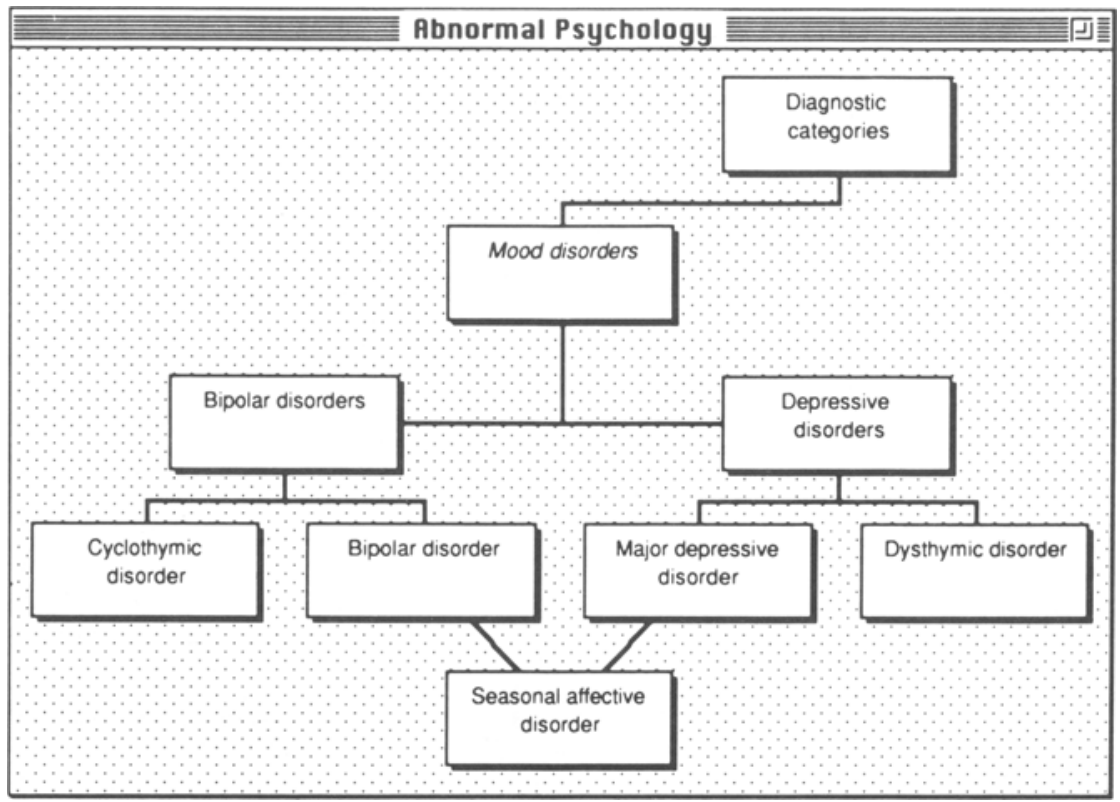

Figure 3. Graphic organizer card for mood disorders.

ing that of one student who verbally reported, "It saved my butt on the last exam." The predominant suggestion for improving the program was that we include examples to supplement definitions.

Of the 17 remaining students, 10 said the main reason they had not used the software was lack of time. Several of these also commented that the lab was generally not open during the hours when they studied. Six said they were satisfied with their performance in the course and had no need for an additional study aid. One student cited discomfort with computers as the primary reason for not using the software.

In order to make the stack more accessible to students on an MS-DOS-dominated campus, an attempt was made to translate the stack into a stand-alone application in the MS-DOS version of Spinnaker Plus. Two problems arose. First, only after purchasing the MS-DOS version of the program did it become apparent that translating stacks from HyperCard to the MS-DOS environment can be accomplished only if both the Macintosh and the MS-DOS versions of the program are purchased. Second, the version of Spinnaker Plus that we acquired was not compatible with HyperCard 2.0, and HyperCard does not allow conversion of stacks from Version 2.0 to earlier versions. Nonetheless, it seems important to pursue a conversion path for the end user portions of the abnormal psychology stack. If it could be placed on the server of the university's LAN, students could then use it on any mouse- equipped MS-DOS machine connected to the LAN. Broader use of the stack by students in the course ought to be achieved under such circumstances.

\section{CONCLUSIONS}

This paper reports on a software development project that is still in progress. The HyperCard authoring shell works well, but the goal of incorporating all concepts taught in a course into a single stack has not yet been achieved. Anecdotal evidence from initial use indicates that some students like the stack as a study aid, but no empirical evidence of its efficacy has been collected. Clearly, much work remains to be done to evaluate the utility of presenting information in this manner.

\section{REFERENCES}

American Psychiatric Association. (1987). Diagnostic and statistical manual of mental disorders (3rd ed., rev.). Washington, DC: Author.

APple Computer. (1991). HyperCard Version 2.1 [computer program]. Cupertino, CA: Author.

DAY, R. S. (1990, November). Outlines versus trees: Same structure, different function. Paper presented at the meeting of the Psychonomic Society, New Orleans.

MoOre, D. W., ReAdence, J. E. (1984). A qualitative review of graphic organizer research. Joumal of Educational Research, 78, 11-17. 